\title{
Efavirenz induces autophagy and aberrant differentiation in normal human keratinocytes
}

\author{
QINGHUA DONG ${ }^{1,2}$, JU-EUN OH ${ }^{1}$, JIN KYU YI $^{1}$, REUBEN H. KIM ${ }^{1,2}$, KI-HYUK SHIN ${ }^{1,2}$, \\ RONALD MITSUYASU ${ }^{2,3}$, NO-HEE PARK ${ }^{1-3}$ and MO K. KANG ${ }^{1,2}$ \\ ${ }^{1}$ School of Dentistry, ${ }^{2}$ Jonsson Comprehensive Cancer Center, ${ }^{3}$ David Geffen School of Medicine, \\ University of California, Los Angeles (UCLA), Los Angeles, CA, USA
}

Received December 4, 2012; Accepted January 8, 2013

DOI: $10.3892 /$ ijmm.2013.1327

\begin{abstract}
Although efavirenz (EFV) is efficacious as an antiretroviral therapy when combined with other antiretroviral drugs, it may cause adverse clinical effects, including skin and mucosal eruptions, central nervous system complications, hepatotoxicity, renal failure and pulmonary complications. The present study investigated the phenotypic alterations caused by EFV in normal human keratinocytes (NHKs) and determined the cell death pathways leading to the lack of epithelial proliferation and regeneration. Replication kinetics, cellular morphology, and protein and mRNA levels of cell cycle regulatory genes and cell death markers were compared between the EFV-exposed cells and the untreated control. EFV treatment led to cell proliferation arrest and cell death of the NHKs by inducing autophagy mediated by proteasome-dependent degradation of p53. EFV also reduced the levels of mTOR and active ERK signaling in NHKs. Chemical inhibition of p53 degradation with a proteasome inhibitor led to reduced autophagic response of NHKs to EFV. In addition, EFV triggered terminal differentiation of NHKs by inducing the expression of involucrin, filaggrin, loricrin and genes involved in cornified envelope formation. Inhibition of autophagy in the EFV-treated NHKs with 3-methylalanine reduced the levels of involucrin and the extent of cell death. Our data indicate that EFV elicits cytotoxic effects on NHKs in part through induction of autophagy and aberrant differentiation of cells.
\end{abstract}

\section{Introduction}

While highly active antiretroviral therapy (HAART) reduces the morbidity and mortality of patients infected with the

Correspondence to: Professor Mo K. Kang, School of Dentistry, Center for the Health Sciences, Room 43-009, University of California, Los Angeles (UCLA), 10833 Le Conte Ave, Los Angeles, CA 90095, USA

E-mail:mkang@dentistry.ucla.edu

Key words: efavirenz, autophagy, keratinocytes, differentiation, p53 human immunodeficiency virus (HIV) (1), prior studies report adverse drug reactions that may alter the course of the antiviral therapy. Various cutaneous and mucosal lesions may result from the use of reverse transcriptase inhibitors (RTIs), as well as protease inhibitors (2). For example, adverse skin reactions including rash, urticaria, erythema multiforme, toxic epidermolysis or Stevens-Johnson syndrome (SJS) have been associated with HAART (3). Similarly, mucosal epithelial lesions in the oral cavity include epithelial desquamation, exfoliative cheilitis, cracks, ulceration and fissure formation (4). These cutaneous and mucosal lesions may result from the use of RTIs, such as azidovudine (AZT), didanosine and efavirenz (EFV), as well as protease inhibitors (2). EFV is one of the commonly used drugs in HAART and is the first medication approved for once-daily dosing (5). Despite its antiviral efficacy at a therapeutic dose, EFV has been linked to skin lesions $(3,6,7)$. In addition, EFV can sometimes cause severe hepatitis, central nervous system (CNS) complications, renal failure and pulmonary complications (8-11). Although these adverse effects may be viewed as hypersensitivity, direct phenotypic and genetic effects of RTIs at the cellular level have not been investigated.

Epithelial tissue regeneration can be hampered by several cell death pathways, including apoptosis, terminal differentiation, cellular senescence and autophagy. Genotoxic signals trigger premature senescence or terminal differentiation in human keratinocytes (12). Oncogene-induced senescence (OIS) is a well-characterized epigenetic phenomenon to halt cellular transformation (13). A recent report showed that mitotic arrest in OIS is mediated by autophagy, a metabolic program leading to catabolic processing of self proteins and organelles (14). Autophagic cell death is characterized by numerous autophagosomes and degradation of cytosolic proteins, whereas the nucleus remains intact until the late stage of cell death (15). This process is orchestrated by multiple protein factors, most notably the autophagy-essential proteins (ATGs), which may exceed 30 different proteins (16). Autophagy can be identified by degradation and lipidation of light chain 3 (LC3), which becomes incorporated into the membrane of autophagosomes and autophagolysosomes until it is degraded (17). Autophagy can be suppressed by chemical inhibitors of PI3K, such as 3-methylalanine (3-MA), and induced by rapamycin (18). 
In the present study, we investigated the cellular effects of EFV on normal human keratinocytes (NHKs) and the underlying molecular mechanisms. Cultured NHKs exposed to EFV exhibited notable loss of cell viability accompanied by elongated morphological alterations. Assessment of the cell death pathway revealed lack of apoptotic responses but induction of autophagy in NHKs exposed to EFV. Sublethal dose of EFV rapidly induced degradation of p53. p53 degradation by EFV occurred together with a reduced mTOR level and activation of ERK phosphorylation, indicating that EFV triggers the canonical autophagic pathway in NHKs. The EFV-treated cells demonstrated premature terminal differentiation, while this effect was attenuated in cells treated with 3-MA. These data indicate that EFV limits epithelial regeneration by triggering autophagy, in part, through proteasome-dependent degradation of p53.

\section{Materials and methods}

Cells and cell culture. NHKs and normal human fibroblasts (NHFs) were prepared from discarded skin or mucosal tissues according to the methods described elsewhere (19). The discarded tissues were utilized to establish the primary cultures as guided by the UCLA Medical Institutional Review Board (MIRB). Human small intestine epithelial cells (CCL-241) were purchased from the American Type Culture Collection (ATCC, Manassas, VA, USA) and cultured in Hybri-Care Medium (ATCC) with 10\% FBS and $30 \mathrm{ng} / \mathrm{ml}$ human EGF (Sigma, St. Louis, MO, USA). HaCaT cells represent a spontaneously immortalized human keratinocyte cell line (20), and OFK6/T cells are oral keratinocytes immortalized with the hTERT gene (21). These cells were maintained in Keratinocyte Growth Medium (Lonza, Walkersville, MD, USA). Peripheral blood mononuclear cells (PBMCs) and Jurket cells were obtained from Dr Anahid Jewett (UCLA School of Dentistry, Los Angeles, CA) and cultured in RPMI-1640 (Invitrogen) supplemented with $10 \%$ FBS. Cell viability was determined by MTT assay after a 48-h exposure to EFV according to the manufacturer's guidelines (ATCC). Replication kinetics was determined by calculating the population doubling (PD) levels according to the methods described elsewhere (19).

Organotypic reconstructs were established using NHKs (22). EFV $(10 \mu \mathrm{M})$ was added to the culture medium at the time of airlifting for 7 days until harvested by fixing in $10 \%$ buffered formalin. Subsequently, H\&E staining was performed on thick $(6 \mu \mathrm{m})$ sagittal sections. Consecutive sections of the same specimens were used for immunohistochemistry (IHC) for proliferating cell nuclear antigen (PCNA) and K1.

Assay of apoptotic cell death. NHKs seeded on 96-well plates were exposed to $10 \mu \mathrm{M}$ EFV for $72 \mathrm{~h}$. DNA fragmentation was determined by staining the cells and TUNEL assay using the In Situ Cell Death detection kit (Roche, South San Francisco, CA, USA). Apoptosis was also detected by western blotting for caspase-3 and poly-ADP-ribose polymerase (PARP) using the cells exposed to EFV and Jurkat cells exposed to ionizing radiation (IR).

Western blotting. Whole cell extracts $(50 \mu \mathrm{g})$ were separated on $4-20 \%$ SDS-PAGE gel and transferred onto Immobilon protein membranes (Millipore, Billerica, MA, USA). The membranes were incubated successively with the primary and the secondary antibodies. Signals were detected using ECL Western blotting detection reagents (Amersham Pharmacia Biotech, Piscataway, NJ, USA).

Reverse transcription and real-time PCR. Total RNA was isolated from cultured cells using the RNeasy Mini kit (Qiagen, Valencia, CA, USA). cDNA was synthesized from $5 \mu \mathrm{g}$ RNA using the Superscript first-strand synthesis system (Invitrogen). We used $1 \mu \mathrm{l}$ cDNA for qPCR amplification using SYBR-Green I Master Mix (Roche). The primer sequences were obtained from the Universal Probe Library (Roche). PCR amplification was performed on LightCycler 480 (Roche). Second derivative $\mathrm{Cq}$ value determination method was used to compare fold-differences according to the manufacturer's instructions (Roche).

Indirect in situ immunostaining. NHKs were exposed to $10 \mu \mathrm{M}$ EFV for $48 \mathrm{~h}$, fixed in $3.7 \%$ formaldehyde for $15 \mathrm{~min}$, and permeabilized in $0.25 \%$ Triton X-100 in PBS for $10 \mathrm{~min}$. Mouse monoclonal anti-p53 or $\alpha$-tubulin and Alex Fluor ${ }^{\circledR} 488$ goat anti-mouse IgG (Invitrogen) were used as primary and secondary antibodies, respectively. Cells were then counterstained with Hoechst $33342(3.3 \mu \mathrm{g} / \mathrm{ml})$. Images were obtained using a Nikon fluorescence microscope. NHKs were treated with $10 \mu \mathrm{M}$ EFV in the absence or presence of $5 \mathrm{mM}$ 3-methylalanine (3-MA) for $48 \mathrm{~h}$. Indirect immunoperoxidase staining (IPS) was performed for involucrin as described previously (23).

\section{Results}

EFV inhibits cell proliferation of NHKs in a cell type-specific manner. To determine the effects of EFV on NHKs, we performed a cell viability assay in cultures exposed to EFV at varying doses from 0 to $40 \mu \mathrm{M}$. After $48 \mathrm{~h}$, we found a dose-dependent reduction in cell viability with the $\mathrm{IC}_{50}$ at $\sim 10 \mu \mathrm{M}$. As a negative control, we included $0.1 \%$ dimethylsulfoxide (DMSO). In contrast, EFV exposure conferred no cytotoxic effects on other cell types, including NHFs, PBMCs, or CCL-241 cells (Fig. 1A). We determined the long-term effects of EFV by serial subculture of primary NHKs in the presence of EFV (Fig. 1B). At $10 \mu \mathrm{M}$ EFV, NHKs completely lost their viability within 10-15 days of exposure, while EFV at 1 and $5 \mu \mathrm{M}$ allowed the cells to undergo continued proliferation. Notably, the cells exhibited extended lifespan at these lower doses, completing PD 20 and 19 at 1 and $5 \mu \mathrm{M}$ $\mathrm{EFV}$, respectively, while the untreated cells senescenced after PD 17. We performed western blot analysis for the proteins involved in cell cycle regulation, such as p53, p16 ${ }^{\mathrm{NK} 4 \mathrm{~A}}, \mathrm{p} 27^{\mathrm{KIP} 1}$ and PCNA in the NHKs following exposure to $10 \mu \mathrm{M} \mathrm{EFV}$ (Fig. 1C). After 1 day of EFV treatment, an increase in $\mathrm{p} 27^{\mathrm{KIP} 1}$ expression and a drastic loss of p53 were noted, while the level of p16 ${ }^{\text {INK4A }}$ did not change. PCNA expression was reduced by $\mathrm{EFV}$, reflecting reduced cell proliferation. Acute exposure to $10 \mu \mathrm{M}$ EFV led to marked changes in cellular morphology characteristic of terminally differentiated keratinocytes, while NHFs remained unchanged (Fig. 1D). We exposed the 3 dimensional (3D) organotypic culture of NHKs to EFV at 
A

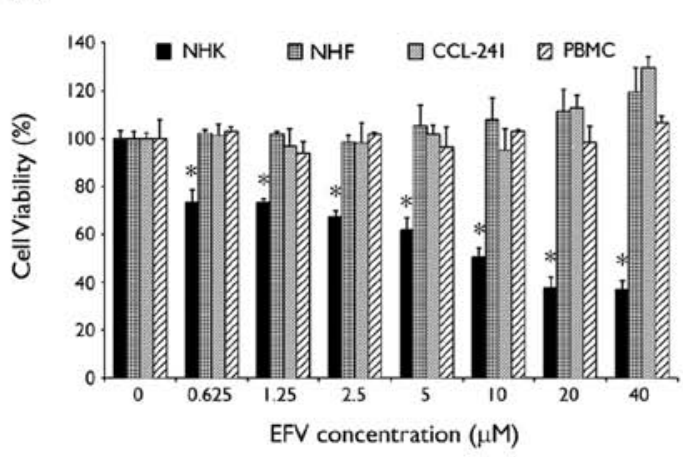

B

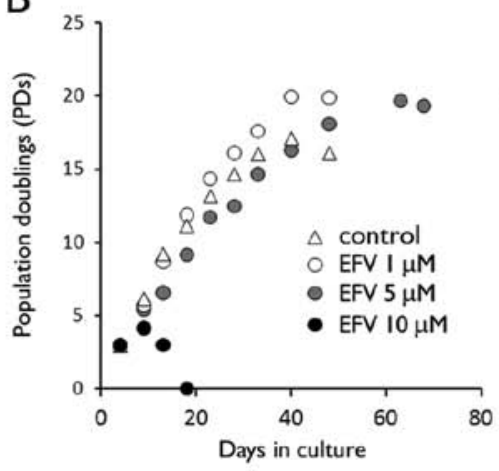

C

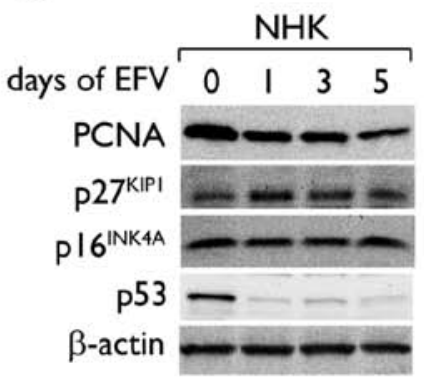

D

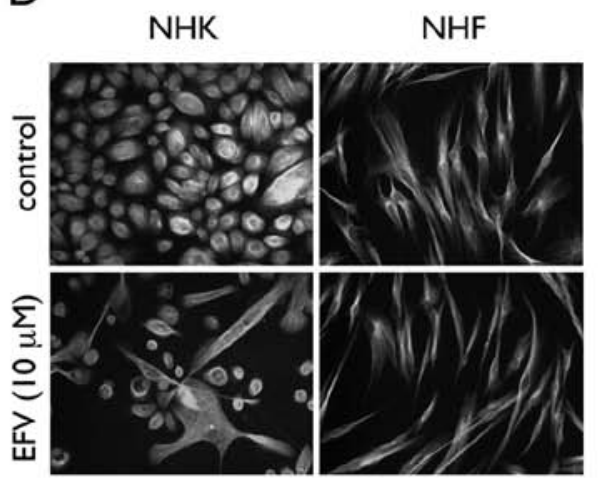

E

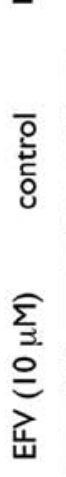

H\&E

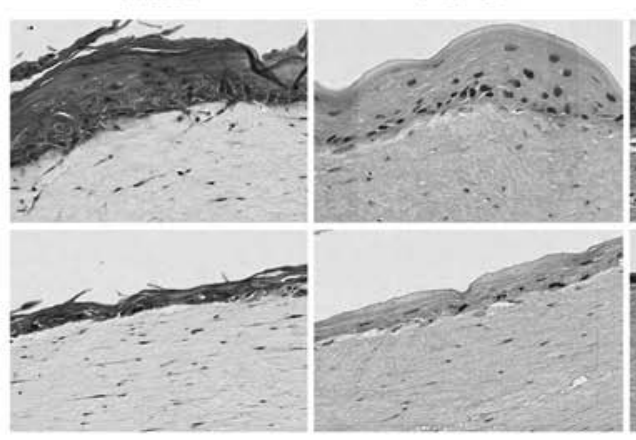

$\mathrm{KI}$

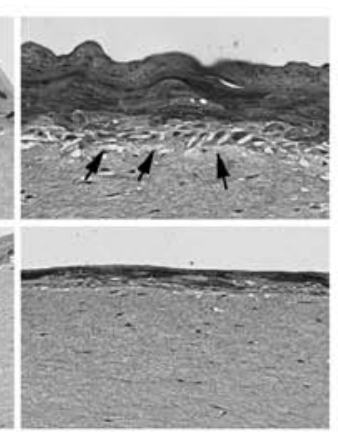

Figure 1. EFV exhibits cytotoxic effects on NHKs in a cell type-specific manner. (A) Cells were exposed to EFV and subjected to MTT assay after 48 h. Error bars, SD. " $\mathrm{P}<0.05$ against control. (B) Replication kinetics is shown in population doublings (PDs) (mean values of at least 3 different counts) against time. (C) NHKs were treated with $10 \mu \mathrm{M}$ EFV, and western blotting was performed for PCNA, p27 ${ }^{\mathrm{KIP1}}$, p16 ${ }^{\mathrm{INK} 4 \mathrm{~A}}$ and p53. (D) NHKs and NHFs were treated with $10 \mu \mathrm{M}$ EFV for $48 \mathrm{~h}$. Immunofluorescence of $\alpha$-tubulin is shown with Hoechst 33342 . Original magnification, x100. (E) Organotypic cultures were maintained with or without EFV $(10 \mu \mathrm{M})$ for 7 days. H\&E staining and IHC for PCNA and K1 are shown. Arrow, undifferentiated (K1-negative) basal cells. Original magnification, $\mathrm{x} 100$.

$10 \mu \mathrm{M}$ for 7 days and found epithelial atrophy, with reduced proliferating and undifferentiated basal cell content, following exposure to EFV (Fig. 1E). However, NHFs in the dermalequivalent layer remained viable and unaltered following EFV exposure. These results demonstrate the cytotoxic and growth inhibitory effects of EFV in a cell type- and dose-dependent manner.

EFV-induced cell death in NHKs involves autophagy but not apoptosis. We explored the hypothesis that apoptosis is induced by EFV in NHKs. We performed terminal deoxynucleotidyl transferase-mediated dUTP nick end-labeling (TUNEL) assay to check for DNA fragmentation in NHKs treated with $10 \mu \mathrm{M}$ EFV for 3 days. As a comparison, we included NHKs exposed to $10 \mu \mathrm{M}$ cisplatin, which induces apoptosis by causing DNA damage (24). Approximately $60 \%$ of the culture showed positive TUNEL staining after cisplatin treatment, while positive TUNEL staining was not detected in the control untreated cells and those exposed to EFV (Fig. 2A). EFV treatment did not cause TUNEL-positive staining in the NHKs. Western blotting was performed for detection of cleaved caspase-3 and PARP, both of which are markers of apoptotic events (25). EFV treatment did not cause caspase-3 activation or cleavage of PARP (Fig. 2B). NHKs treated with EFV showed no change in the level of caspase-3, but almost complete loss of full-length PARP. However, this was not consistent with the apoptotic response as evinced in Jurkat cells exposed to 5 Gy IR, which showed accumulation of the cleaved PARP, reflecting activation of caspase- 3 . Notably, EFV treatment led to a notable reduction in the levels of CDK2 and cyclin A2 (Fig. 2C), which are required for entry into mitosis (26), suggesting potential arrest of the cell cycle in the $\mathrm{S}$ phase. Hence, the cell death noted in NHKs following exposure to EFV did not involve apoptosis.

Next, we examined the occurrence of autophagy, known as an alternative pathway of programmed cell death (27). We assessed the levels of LC3-I and LC3-II, which represent the parent form and the cleaved form, respectively. Following 3 days of EFV treatment, we noted an increased level of LC3-II, while this was abolished by co-treatment with 3-MA (Fig. 3A). As a control, NHKs were exposed to rapamycin, which induces the level of LC3-II. EFV treatment almost completely abolished the level of Bcl-2 by day 1. There was a time-dependent increase in the levels of Beclin-1 and ATG5, and this paralleled the increase in LC3-II in the NHKs following treatment with EFV (Fig. 3B). The LC3-II level did not change in the NHFs exposed to $\mathrm{EFV}$, whereas rapamycin led to a notable induction in LC3-II (Fig. 3C). These data suggest that EFV induces autophagy in NHKs but not in NHFs, and this may explain the cell type-specific cytotoxicity of EFV. 

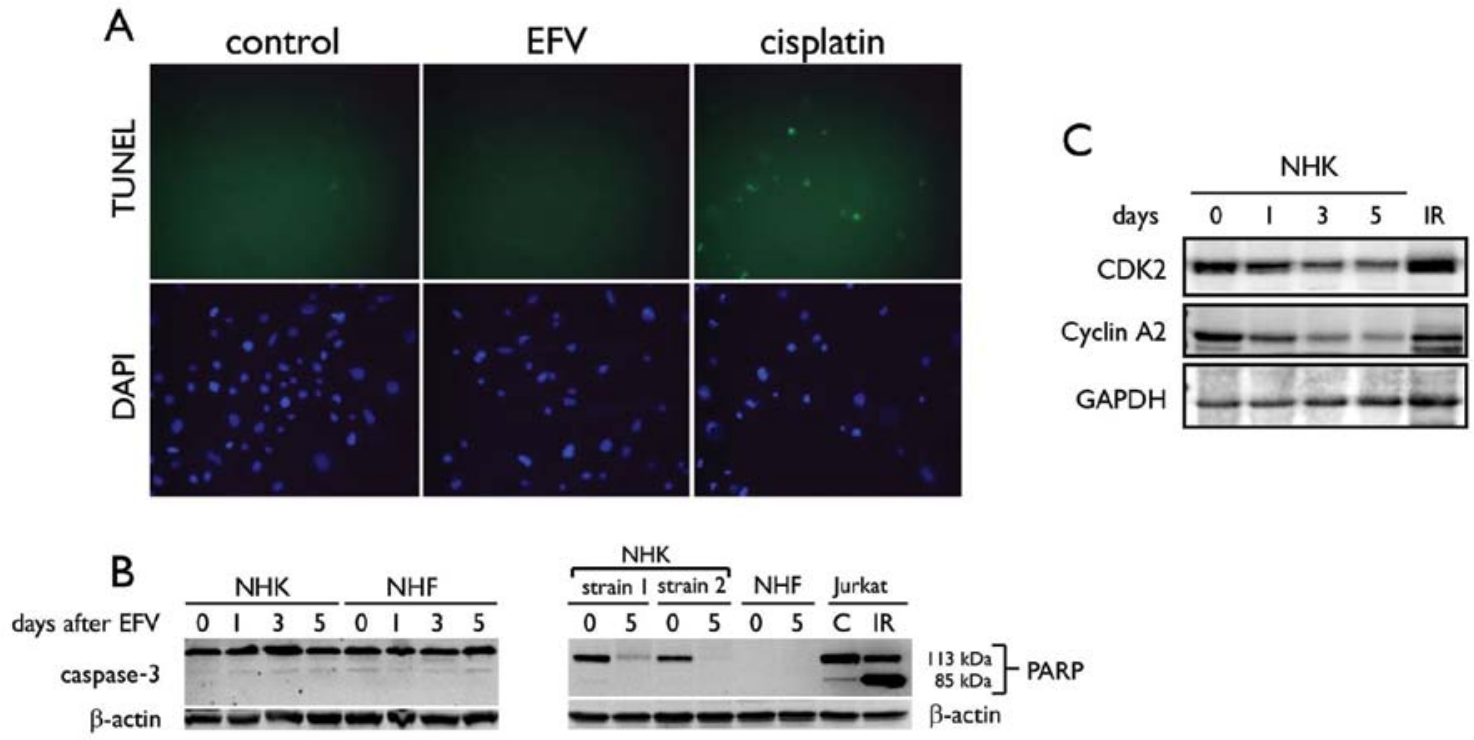

Figure 2. EFV does not induce apoptosis in NHKs. (A) NHKs were exposed to $10 \mu \mathrm{M} \mathrm{EFV} \mathrm{for} 72 \mathrm{~h}$ and subjected to TUNEL assay to detect fragmentation of DNA ends, characteristic of apoptotic cell death. Cells treated with $10 \mu \mathrm{M}$ cisplatin for $24 \mathrm{~h}$ were used as a positive control. (B) NHKs and NHFs exposed to $10 \mu \mathrm{M} \mathrm{EFV}$ were assayed by western blotting to detect cleavage of caspase-3 and PARP. (C) Western blotting showed progressive reduction of CDK2 and cyclin A2 in NHKs exposed to $10 \mu \mathrm{M}$ EFV. As a control, we included Jurkat cells exposed to 5 Gy ionizing radiation (indicated by IR). $\beta$-actin and GAPDH served as loading controls.

A

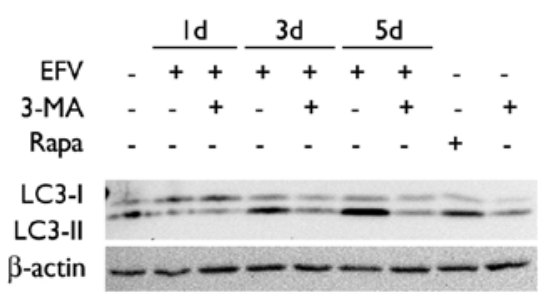

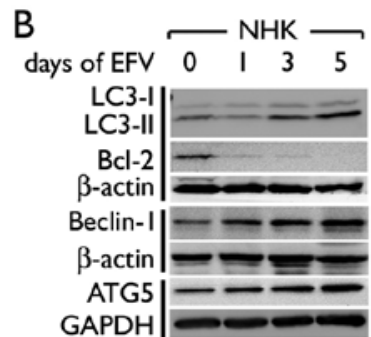

C

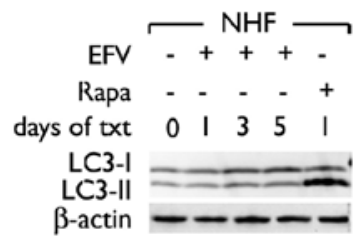

Figure 3. EFV induces autophagy in NHKs. (A) NHKs were exposed to $10 \mu \mathrm{M}$ EFV with or without 3-MA. Levels of LC3-I and LC3-II were determined by western blotting. (B) Levels of LC3-I, LC3-II, Bcl-2, Beclin-1 and ATG5 were determined by western blotting. (C) NHFs exposed to $10 \mu$ M EFV were assayed for the levels of LC3-I and LC3-II by western blotting. The cells treated with rapamycin (100 nM) (Rapa) were used as a positive control. $\beta$-actin or GAPDH served as a loading control. Days of txt, days of drug treatment.

EFV stimulates proteosome-dependent degradation of p53, loss of mTOR, and activation of ERK1/2 in NHKs. Previous studies have demonstrated that cytoplasmic p53 plays a causative role in autophagy (28). Western blotting showed that EFV treatment led to a rapid reduction in p53 in NHKs (Fig. 4B). This was confirmed by immunofluorescence staining of $\mathrm{p} 53$, which showed loss of nuclear and cytoplasmic p53 in the EFV-treated cells (Fig. 4A). Since p53 undergoes ubiquitin-dependent proteasomal degradation, we exposed the cells to EFV in the presence of MG132, a proteasome inhibitor. EFV treatment led to a reduction in the p53 level and a strong increase in LC3-II accumulation, reflecting induction of autophagy (Fig. 4B). MG132 blocked the EFV-mediated p53 degradation and notably reduced the level of LC3-II in the cells exposed to EFV. On the contrary, co-treatment of 3-MA, which suppressed EFV-mediated autophagy in NHKs, did not inhibit p53 degradation in the cells exposed to EFV (Fig. 4C), suggesting that p53 degradation does not result from EFV-mediated autophagy. The EFV-treated NHKs showed reduced phosphorylation of mTOR (Ser2448), similar to the cells treated with rapamycin (Fig. 4D). This occurred together with a reduced protein level of mTOR. In contrast, the azidothymidine (AZT)-treated cells exhibited no changes in mTOR phosphorylation. EFV treatment also led to phosphorylation of ERK1/2 (Thr202 and Tyr204) while other treatments showed no effect. The above data suggest that EFV-mediated autophagy in NHKs occurs with loss of p53 and $\mathrm{mTOR}$, and activation of the ERK pathway.

Autophagy induced by EFV is linked with terminal differentiation of NHKs. Prior to cell death, the EFV-treated cells exhibited morphological changes resembling terminal differentiation (Fig. 1D). This was confirmed by western blotting for the markers of keratinocyte differentiation, i.e. involucrin, filaggrin and loricrin. The levels of these markers increased in NHKs exposed to EFV in a timedependent manner (Fig. 5A). After 5 days of EFV exposure, involucrin expression was increased to a level similar to calcium treatment (data not shown), which triggers terminal differentiation (19). EFV-induced keratinocyte differentiation 
A
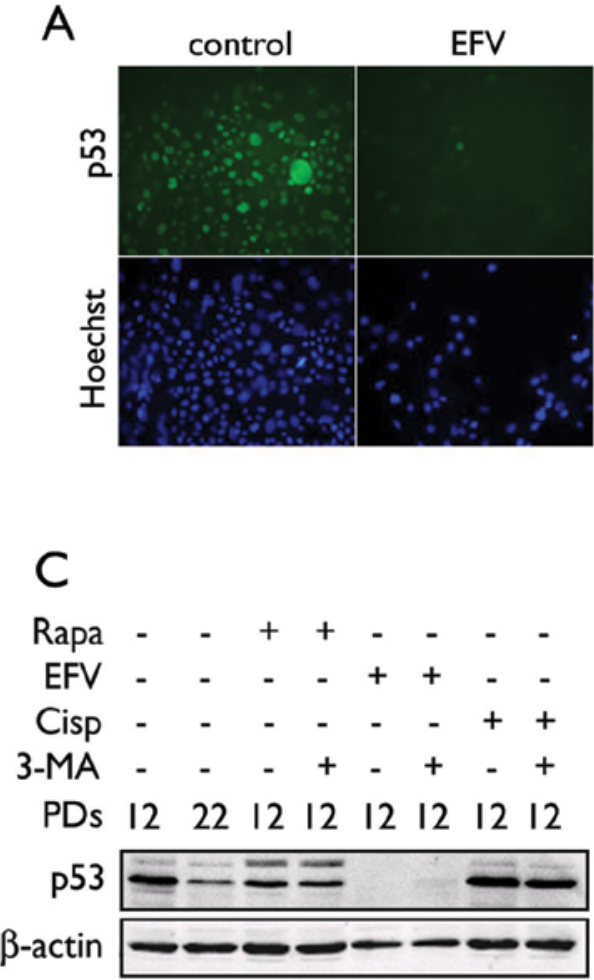

B
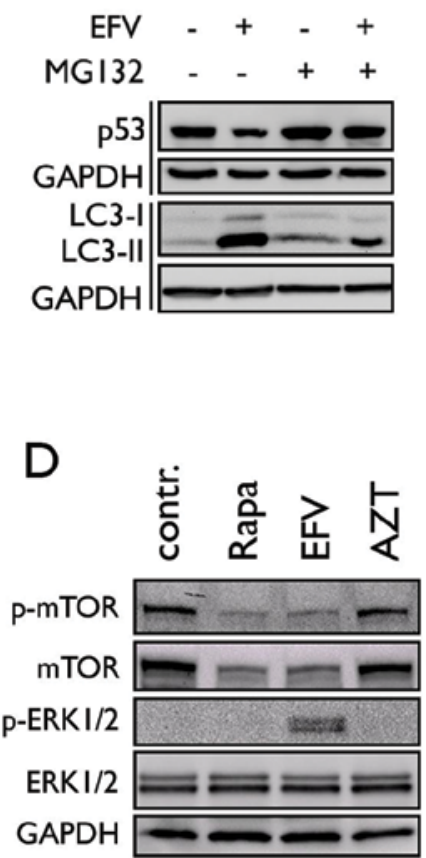

Figure 4. EFV causes proteosome-dependent degradation of p53. (A) NHKs exposed to $10 \mu \mathrm{M}$ EFV for $48 \mathrm{~h}$ were stained for $\mathrm{p} 53$ by indirect immunofluorescence staining and Hoechst 33342 counterstain. Original magnification, $\mathrm{x} 100$. (B) NHKs were exposed to $10 \mu \mathrm{M}$ EFV for $48 \mathrm{~h}$ with or without the addition of $10 \mu \mathrm{M}$ MG132 $2 \mathrm{~h}$ prior to collection. Western blotting was performed for p53 and LC3. (C) NHKs at PD 12 were exposed to $10 \mu \mathrm{M}$ EFV for $48 \mathrm{~h}, 100 \mathrm{nM}$ rapamycin (Rapa) for 5 days or $10 \mu \mathrm{M}$ cisplatin (Cisp) for $24 \mathrm{~h}$ in the absence and presence of 3-MA (5 mM). The p53 level was determined by western blotting. (D) NHKs were exposed to EFV, rapamycin (Rapa), or AZT, and the cells were assayed for phosphorylated and total mTOR, and phosphorylated and total ERK1/2. $\beta$-actin or GAPDH served as a loading control.

A

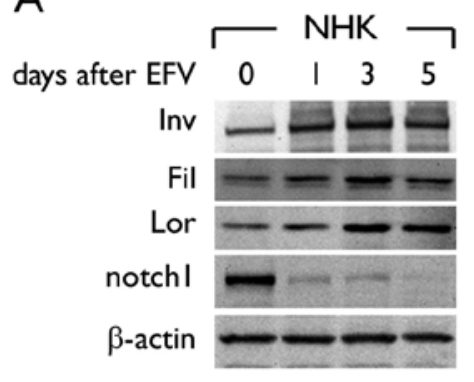

B

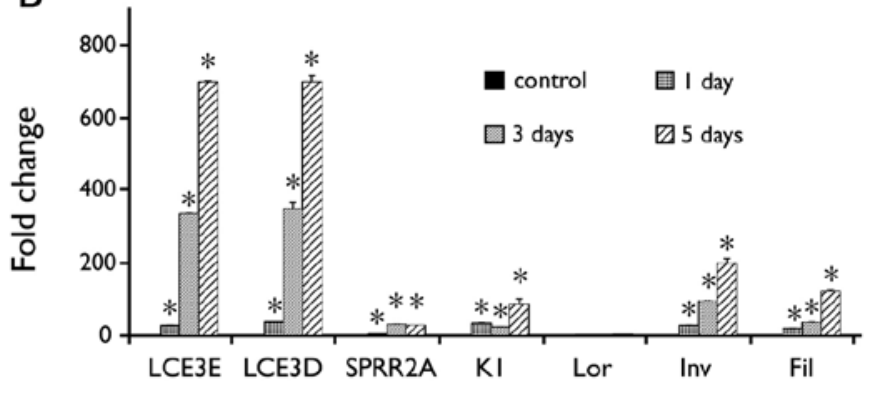

D

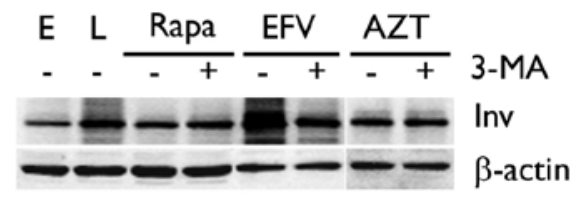

Figure 5. EFV induces terminal differentiation in NHKs. (A) Western blotting was performed with EFV-treated NHKs to detect various proteins as indicated. (B) mRNA expression levels for differentiation-associated genes were determined by qPCR. Error bars, SD. ${ }^{*} \mathrm{P}<0.05$ against control. (C) NHKs were exposed to $10 \mu \mathrm{M}$ EFV for $48 \mathrm{~h}$ with or without 3-MA $(5 \mathrm{mM})$ and stained for involucrin by IPS. Original magnification, x100. (D) Involucrin expression was determined in NHKs exposed to rapamycin (Rapa), EFV or AZT in the absence or presence of 3-MA. E, proliferating cells at PD 12; L, senescent cells at PD 22 were included as controls. $\beta$-actin served as a loading control. Inv, involucrin; Fil, filaggrin; Lor, loricrin, K1, keratin 1.

occurred with loss of the Notch1 protein level after exposure to EFV. We confirmed the induction of the genes involved in keratinocyte differentiation by qPCR. All tested genes, LCE3E, LCE3D, SPRR2A, keratin 1, loricrin, involucrin and 
filaggrin, were progressively induced at the mRNA expression level by EFV treatment (Fig. 5B). In situ immunoperoxidase staining revealed the increased intensity of involucrin staining and lack of cell proliferation in NHKs exposed to EFV (Fig. 5C). However, when the cells were co-treated with EFV and 3-MA, there was a reduction in involucrin staining and partial recovery of replicating and surviving cells. Western blotting revealed that EFV strongly induced the involucrin level beyond that of senescent NHKs at late-passage (PD 22), and 3-MA treatment notably reduced the involucrin protein level in the EFV-treated cells (Fig. 5D). Notably, rapamycin or AZT did not trigger keratinocyte differentiation. Thus, EFV-mediated autophagy in the NHKs was uniquely linked with aberrant keratinocyte differentiation, which is partially responsible for the cytotoxic effects of the drug.

\section{Discussion}

Exposure of NHKs to EFV led to autophagic cell death associated with terminal differentiation. This was demonstrated by rapid loss of cell proliferation and viability that accompanied induction of LC3-II and the markers of keratinocyte terminal differentiation in cells exposed to EFV. Organotypic culture study showed that EFV treatment eliminates the undifferentiated and proliferating cells in the epithelium. This leads to lack of epithelial regeneration and causes atrophy. Therefore, aberrant differentiation of keratinocytes caused by EFV would, at least in part, be responsible for cutaneous adverse effects noted in patients. These phenotypic responses occurred uniquely in NHKs but not in other cell types, i.e. fibroblasts, intestinal cells or leukocytes. The 3D tissue reconstruction model revealed epithelial atrophy and the lack of basal cell proliferation and tissue regeneration upon exposure to EFV, while NHFs in the dermal equivalent layer remained unchanged. This cell type-specificity was paralleled by induction of autophagy in NHKs but not in NHFs, indicating that the cytotoxicity of EFV is linked with autophagy. On the contrary, induction of apoptosis was not noted in EFV-treated cells. We also tested several other genotoxic agents, such as actinomycin D, dexamethasone, etoposide and methyl-nitro-nitrosoguanine (data not shown). Apoptotic signaling from these strong genotoxic agents was extremely weak or absent in NHKs. Likewise, we recently reported that IR failed to trigger apoptosis in NHKs, while it strongly induced apoptosis in Jurkat cells (29). Thus, apoptosis was poorly induced in these cells.

EFV treatment increased the protein level of Beclin-1, ATG5 and p27 ${ }^{\mathrm{KIP} 1}$, and drastically reduced p53 and Bcl-2. The increased $\mathrm{p} 27^{\mathrm{KIP} 1}$ level may have resulted from autophagy through the AMP-activated protein kinase pathway (30). Loss of Bcl-2 may allow Beclin-1 binding to $\mathrm{hVps} 34$, a class III PI3K (31), thereby promoting autophagy in the cells treated with EFV. EFV also led to marked reduction in cyclin A2, which is required for mitotic entry (26), suggesting possible $\mathrm{S}$ phase arrest in the treated cells. Loss of cyclin A2 may have contributed to the growth inhibitory effects of EFV on NHKs. Autophagy occurs along with proteosome-dependent degradation of p53; specific inhibition of p53 is sufficient to trigger autophagic cell death (28). p53 degradation in the EFV-treated NHKs occurred quite rapidly in a proteosome-dependent manner, and blockage of the p53 degradation notably reduced the autophagic response to EFV. p53 degradation did not occur in NHFs, which showed no phenotypic changes to EFV (data not shown). We previously showed that the levels of PCNA, involucrin, p16 ${ }^{\mathrm{INK} 4 \mathrm{~A}}$ and $\mathrm{p} 53$ do not change in exponentially replicating cells until the cells approach replicative senescence $(32,33)$. In the present study, the inhibitory effect of EFV was noted primarily on $\mathrm{p} 53$, while that of $\mathrm{p} 16^{\mathrm{INK} 4 \mathrm{~A}}$ was not evident (Fig. 1C). In addition, we used rapidly proliferating cells for the experiments. Thus, the possibility that the loss of p53 occurred through replicative senescence during EFV exposure is very remote. These data suggest that p53 degradation in NHKs may have a causative role in autophagy in response to $\mathrm{EFV}$.

Autophagy is often linked with a variety of other cellular processes, such as senescence, ER stress, or differentiation. A recent study showed that autophagy mediates the mitotic arrest during OIS and demonstrated the interdependence between the two processes (14). Our data showed that autophagy was linked to terminal differentiation in NHKs as a mechanism of cell death. The EFV-treated NHKs demonstrated elongated morphology in culture and strongly expressed the markers of keratinocyte differentiation. These phenotypic changes including cell death were partially inhibited by 3-MA, an inhibitor of the PI3K pathway (18). Rapamycin also induced autophagy in NHKs but did not trigger differentiation, suggesting that the mechanisms causing autophagy were different between rapamycin and EFV. Keratinocyte differentiation is regulated by the notch signaling pathway (34). However, EFV-induced differentiation occurred without notch induction. Rather, the notch protein level rapidly decreased in cells exposed to EFV, presumably due to p53 degradation since p53 is required for notch expression (34).

Although autophagy is a method of programmed cell death and can function as a tumor-suppressive mechanism, it may also lead to cell survival under stressful conditions. Beclin-1 was found to be deleted in large portions (50-75\%) of various types of human cancers, including breast and ovarian $(35,36)$. Introduction of beclin-1 into a cancer cell line led to autophagy and loss of cell proliferation and in vivo tumorigenicity (37). A subsequent study showed that monoallelic deletion of beclin-1 in a mouse model led to increased tumorigenesis associated with reduced autophagy (38). These studies suggest the tumor suppressive effects of autophagy. In contrast, autophagy does play a role in maintaining the viability of highly proliferative cancer cells, particularly in the center of the tumor mass where the cells are under severe metabolic stress. A study by Degenhardt et al (39) demonstrated induction of autophagy in regions of metabolic stress to mitigate the ischemic cell death. Furthermore, autophagy was found to be induced in leukemic cells undergoing anticancer therapy with a histone deacetylase inhibitor, such as suberoylanilide hydroxamic acid (SAHA), as a protective and survival mechanism (40). Chemical and genetic disruption of autophagy led to enhanced anticancer efficacy of SAHA in the present study. Therefore, autophagy may indeed be a mechanism by which cancer cells gain resistance to chemotherapeutic agents or protect cancer cells from metabolic stress.

In $\mathrm{HIV}^{+}$patients undergoing long-term therapeutic exposure to EFV, the occurrence of autophagy and its contri- 
bution to tumorigenesis need to be investigated. The mean plasma concentration of EFV in $\mathrm{HIV}^{+}$patients under the antiretroviral regimen was found to be $8.7 \mu \mathrm{M}$ for those who responded to therapy (41). Another study showed the mean plasma concentration to be $6.9 \mu \mathrm{M}$ with a wide range from 0.4 to $48 \mu \mathrm{M}$, and suggested an effective therapeutic plasma concentration of EFV at 3-13 $\mu \mathrm{M}$ (42). Thus, $10 \mu \mathrm{M}$ EFV used in the present study was within the clinically relevant concentration at which EFV exhibits an antiviral effect. It is possible that EFV-induced autophagy has contrasting effects on cells at different concentrations. As shown in Fig. 1D, EFV caused cell death at $10 \mu \mathrm{M}$ but allowed enhanced cell proliferation at 1 and $5 \mu \mathrm{M}$, extending the replicative lifespan of cells. We also found that chronic exposure of immortalized oral keratinocytes harboring the human papillomavirus (HPV) genome to $5 \mu \mathrm{M}$ EFV interfered with terminal differentiation and led to a transformed phenotype (data not shown). Although EFV triggered terminal differentiation and autophagic cell death in NHKs at $10 \mu \mathrm{M}$, it may have a tumor-promoting effect at a lower concentration by protecting aberrant cells from metabolic stress and suppressing the cell death pathway. These possibilities warrant further investigation.

\section{Acknowledgements}

This study was supported in part by the grants (DE18295 and DE18959) from the National Institute of Dental and Craniofacial Research (NIDCR) and the Jack Weichman Endowed Fund. We thank NIH AIDS Research and the Reference Reagents Program for providing efavirenz.

\section{References}

1. Kalkut G: Antiretroviral therapy: an update for the non-AIDS specialist. Curr Opin Oncol 17: 479-484, 2005.

2. Borrás-Blasco J, Navarro-Ruiz A, Borrás C and Casterá E: Adverse cutaneous reactions associated with the newest antiretroviral drugs in patients with human immunodeficiency virus infection. J Antimicrob Chemother 62: 879-888, 2008.

3. Borrás-Blasco J, Belda A, Rosique-Robles JD, Casterá MD and Abad FJ: Burning mouth syndrome due to efavirenz therapy. Ann Pharmacother 40: 1471-1472, 2006.

4. Casariego Z and Ben G: Oral manifestations of HIV infection in Argentina: a study of 1889 cases. Med Oral 3: 271-276, 1998

5. Porche DJ: Efavirenz. J Assoc Nurses AIDS Care 11: 95-98, 2000.

6. Dona C, Soriano V, Barreiro P, Jiménez-Náchera I and GonzálezLahoz J: Toxicity associated to efavirenz in HIV-infected persons enrolled in an expanded access program. Med Clin 115 337-338, 2000

7. Yoshimoto E, Konishi M, Takahashi K, Murakawa K, Maeda K, Mikasa $\mathrm{K}$ and Yamashina Y: The first case of efavirenz-induced photosensitivity in a Japanese patient with HIV infection. Intern Med 43: 630-631, 2004.

8. Behrens GM, Stoll M and Schmidt RE: Pulmonary hypersensitivity reaction induced by efavirenz. Lancet 357: 1503-1504, 2001.

9. Angel-Moreno-Maroto A, Suárez-Castellano L, HernándezCabrera $M$ and Pérez-Arellano JL: Severe efavirenz-induced hypersensitivity syndrome (not-DRESS) with acute renal failure. J Infect 52: e39-e40, 2006.

10. Punwani K, Suedkamp S, Nguyen D and Song JC: Update on the CNS adverse effects of Sustiva (efavirenz). AIDS Alert 22 32-34, 2007.

11. Brück S, Witte S, Brust J, Schuster D, Mosthaf F, Procaccianti M, Rump JA, Klinker H, Petzold D and Hartmann M: Hepatotoxicity in patients prescribed efavirenz or nevirapine. Eur J Med Res 13: 343-348, 2008.
12. Bertrand-Vallery V, Boilan E, Ninane N, Demazy C, Friguet B, Toussaint O, Poumay Y and Debacq-Chainiaux F: Repeated exposures to UVB induce differentiation rather than senescence of human keratinocytes lacking p16(INK-4A). Biogerontology 11: 167-181, 2010.

13. Agger K, Cloos PA, Rudkjaer L, Williams K, Andersen G, Christensen J and Helin K: The H3K27me3 demethylase JMJD3 contributes to the activation of the INK4A-ARF locus in response to oncogene- and stress-induced senescence. Genes Dev 23: 1171-1176, 2009.

14. Young AR, Narita M, Ferreira M, Kirschner K, Sadaie M, Darot JF, Tavaré S, Arakawa S, Shimizu S, Watt FM and Narita M: Autophagy mediates the mitotic senescence transition. Genes Dev 23: 798-803, 2009.

15. Klionsky DJ: Autophagy: from phenomenology to molecular understanding in less than a decade. Nat Rev Mol Cell Biol 8: 931-937, 2007.

16. Liang C and Jung JU: Autophagy genes as tumor suppressors. Curr Opin Cell Biol 22: 226-233, 2010.

17. Kabeya Y, Mizushima N, Ueno T, Yamamoto A, Kirisako T, Noda T, Kominami E, Ohsumi Y and Yoshimori T: LC3, a mammalian homologue of yeast Apg8p, is localized in autophagosome membranes after processing. EMBO J 19: 5720-5728, 2000

18. Wu YT, Tan HL, Shui G, Bauvy C, Huang Q, Wenk MR, Ong CN, Codogno P and Shen HM: Dual role of 3-methyladenine in modulation of autophagy via different temporal patterns of inhibition on class I and III phosphoinositide 3-kinase. J Biol Chem 285: 10850-10861, 2010.

19. Kang MK, Bibb C, Baluda MA, Rey O and Park NH: In vitro replication and differentiation of normal human oral keratinocytes. Exp Cell Res 258: 288-297, 2000.

20. Boukamp P, Petrussevska RT, Breitkreutz D, Hornung J, Markham A and Fusenig NE: Normal keratinization in a spontaneously immortalized aneuploid human keratinocyte cell line. J Cell Biol 106: 761-771, 1988.

21. Dickson MA, Hahn WC, Ino Y, Ronfard V, Wu JY, Weinberg RA, Louis DN, Li FP and Rheinwald JG: Human keratinocytes that express hTERT and also bypass a p16(INK4a)-enforced mechanism that limits life span become immortal yet retain normal growth and differentiation characteristics. Mol Cell Biol 20: 1436-1447, 2000.

22. Dongari-Bagtzoglou A and Kashleva H: Development of a highly reproducible three-dimensional organotypic model of the oral mucosa. Nat Protoc 1: 2012-2018, 2006.

23. Kang MK, Kim RH, Kim SJ, Yip FK, Shin KH, Dimri GP, Christensen R, Han T and Park NH: Elevated Bmi-1 expression is associated with dysplastic cell transformation during oral carcinogenesis and is required for cancer cell replication and survival. Br J Cancer 96: 126-133, 2007.

24. Kim Y, McBride J, Zhang R, Zhou X and Wong DT: p12 (CDK2AP1) mediates DNA damage responses induced by cisplatin. Oncogene 24: 407-418, 2005 .

25. Kurokawa M and Kornbluth S: Caspases and kinases in a death grip. Cell 138: 838-854, 2009.

26. Gong D and Ferrell J Jr: The roles of cyclin A2, B1, and B2 in early and late mitotic events. Mol Biol Cell 21: 3149-3161, 2010.

27. Bursch W, Ellinger A, Gerner C, Fröhwein U and SchulteHermann R: Programmed cell death (PCD). Apoptosis, autophagic PCD, or others? Ann NY Acad Sci 926: 1-12, 2000.

28. Tasdemir E, Maiuri MC, Galluzzi L, Vitale I, DjavaheriMergny M, D'Amelio M, Criollo A, Morselli E, Zhu C, Harper F, Nannmark U, Samara C, Pinton P, Vicencio JM, Carnuccio R, Moll UM, Madeo F, Paterlini-Brechot P, Rizzuto R, Szabadkai G, Pierron G, Blomgren K, Tavernarakis N, Codogno P, Cecconi F and Kroemer G: Regulation of autophagy by cytoplasmic p53. Nat Cell Biol 10: 676-687, 2008.

29. Dong Q, Oh JE, Chen W, Kim R, Kim RH, Shin KH, McBride WH, Park NH and Kang MK: Radioprotective effects of Bmi-1 involve epigenetic silencing of oxidase genes and enhanced DNA repair in normal human keratinocytes. J Invest Dermatol 131: 1216-1225, 2011.

30. Liang J, Shao SH, Xu ZX, Hennessy B, Ding Z, Larrea M, Kondo S, Dumont DJ, Gutterman JU, Walker CL, Slingerland JM and Mills GB: The energy sensing LKB1-AMPK pathway regulates $\mathrm{p} 27(\mathrm{kip} 1)$ phosphorylation mediating the decision to enter autophagy or apoptosis. Nat Cell Biol 9: 218-224, 2007.

31. Pattingre S, Tassa A, Qu X, Garuti R, Liang XH, Liang XH, Mizushima N, Packer M, Schneider MD and Levine B: Bcl-2 antiapoptotic proteins inhibit Beclin 1-dependent autophagy. Cell 122: 927-939, 2005. 
32. Kang MK, Guo W and Park NH: Replicative senescence of normal human oral keratinocytes is associated with the loss of telomerase activity without shortening of telomeres. Cell Growth Differ 9: 85-95, 1998.

33. Kang MK, Kameta A, Shin KH, Baluda MA and Park NH: Senescence occurs with hTERT repression and limited telomere shortening in human oral keratinocytes cultured with feeder cells. J Cell Physiol 199: 364-370, 2004.

34. Yugawa T, Narisawa-Saito M, Yoshimatsu Y, Haga K, Ohno S, Egawa N, Fujita M and Kiyono T: DeltaNp63alpha repression of the Notch1 gene supports the proliferative capacity of normal human keratinocytes and cervical cancer cells. Cancer Res 70: 4034-4044, 2010.

35. Futreal PA, Söderkvist P, Marks JR, Iglehart JD, Cochran C, Barrett JC and Wiseman RW: Detection of frequent allelic loss on proximal chromosome $17 \mathrm{q}$ in sporadic breast carcinoma using microsatellite length polymorphisms. Cancer Res 52: 2624-2627, 1992.

36. Cliby W, Ritland S, Hartmann L, Dodson M, Halling KC, Keeney G, Podratz KC and Jenkins RB: Human epithelial ovarian cancer allelotype. Cancer Res 53 (Suppl 10): S2393-S2398, 1993.

37. Liang XH, Jackson S, Seaman M, Brown K, Kempkes B, Hibshoosh $\mathrm{H}$ and Levine B: Induction of autophagy and inhibition of tumorigenesis by beclin 1. Nature 402: 672-676, 1999.
38. Qu X, Yu J, Bhagat G, Furuya N, Hibshoosh H, Troxel A, Rosen J, Eskelinen EL, Mizushima N, Ohsumi Y, Cattoretti G and Levine B: Promotion of tumorigenesis by heterozygous disruption of the beclin 1 autophagy gene. J Clin Invest 112: 1809-1820, 2003.

39. Degenhardt K, Mathew R, Beaudoin B, Bray K, Anderson D, Chen G, Mukherjee C, Shi Y, Gélinas C, Fan Y, Nelson DA, Jin S and White E: Autophagy promotes tumor cell survival and restricts necrosis, inflammation, and tumorigenesis. Cancer Cell 10: 51-64, 2006.

40. Carew JS, Nawrocki ST, Kahue CN, Zhang H, Yang C, Chung L, Houghton JA, Huang P, Giles FJ and Cleveland JL: Targeting autophagy augments the anticancer activity of the histone deacetylase inhibitor SAHA to overcome Bcr-Abl-mediated drug resistance. Blood 110: 313-322, 2007.

41. Ståhle L, Moberg L, Svensson JO and Sönnerborg A: Efavirenz plasma concentrations in HIV-infected patients: inter- and intraindividual variability and clinical effects. Ther Drug Monit 26: 267-270, 2004.

42. Marzolini C, Telenti A, Decosterd LA, Greub G, Biollaz J and Buclin T: Efavirenz plasma levels can predict treatment failure and central nervous sytem side effects in HIV-1-infected patients. AIDS 15: 71-75, 2001 